\title{
Fully Automated and Adaptive Intensity Normalization Using Statistical Features for Brain MR Images
}

\author{
Evgin Goceri \\ Akdeniz University, Engineering Faculty, Computer Engineering Department, 07058, Antalya \\ Tel.: 0242-310-4393, evgin@akdeniz.edu.tr \\ Received: 26 January 2018 \\ Accepted: 5 March 2018 \\ DOI: $10.18466 /$ cbayarfbe. 384729
}

\begin{abstract}
Accuracy of the results obtained by automated processing of brain magnetic resonance images has vital importance for diagnosis and evaluation of a progressive disease during treatment. However, automated processing methods such as segmentation, registration and comparison of these images are challenging issues. Because intensity values do not only depend on the underlying tissue type. They can change due to scanner-related artifacts and noise, which usually occurs in magnetic resonance images. In addition to intensity variations, low contrast and partial volume effects increases the difficulty in automated methods with these images. Intensity normalization has a significant role to increase performance of automated image processing methods. Because it is applied as a preprocessing step and efficiency of the other steps in these methods is based on the results obtained from the preprocessing step. The goal of intensity normalization is to make uniform the mean and variance values in images. Different methods have been applied for this purpose in the literature and each method has been tested with different kind of images. In this work; 1) The state-of-art normalization methods applied for magnetic resonance images have been reviewed. 2) A fully automated and adaptive approach has been proposed for intensity normalization in brain magnetic resonance images. 3) Comparative performance evaluations of the results obtained by four different normalization approaches using the same images have been presented. Comparisons of all methods implemented in this work indicate a better performance of the proposed approach for brain magnetic resonance images.
\end{abstract}

Keywords: Brain MR images, Gaussian filtering, homomorphic filtering, intensity normalization, local normalization

\section{Introduction}

Automated processing of brain Magnetic Resonance (MR) images is a challenging issue due to intensity variations caused by scanner-related artifacts, noise and partial volume effects. Unclear edges between brain tissues, which are Cerebrospinal Fluid (CSF), Gray Matter (GM), White Matter (WM) (particularly between GM and WM), and low-contrast increase this difficulty. An example brain MR image is presented in Figure 1.

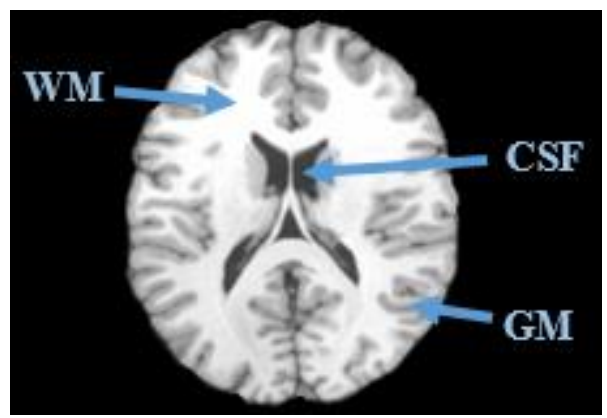

Figure 1. Brain MR image and tissues
Intensity normalization is applied as an initial step to increase performance of automated image processing techniques. Because, the goal of intensity normalization is to make uniform the mean and variance of intensity values in images. Intensity normalization has an important influence in registration [1], longitudinal segmentation [2], cross-sectional segmentation [3], longitudinal quantification [4] and other measures [5,6]. Intensity normalization is also important for MR image comparison, which is usually required for disease diagnosis and evaluation of a progressive disease during treatment period. MR image datasets, which are taken at different times from the same patient, are usually compared to detect or quantify the changes in tissues. The time series analysis gives information about the stage or progression of the disease $[7,8]$.

In this work, intensity normalization techniques applied with MR images have been reviewed. It has been observed that Gaussian filtering [9-14] and homomorphic filtering 
have been mostly used [15-22]. In addition to these methods, there are some deterministic and probabilistic approaches applied with different image modalities in the literature (Section 2). Also, in this work, a Fully Automated and Adaptive Intensity Normalization (FAIN) has been proposed for brain MR images. FAIN, which is an improved version of a recently published work [23], is a probabilistic approach. An important drawback of the method given in [23] is that users have to define three regions manually on brain images. However, users may define those regions with different sizes, which may cause undesired results. Because the size of a region has to be large enough (at least $20 \times 20$ pixels) to generate statistical information and obtain efficient results (Figure 2). Also, location of those regions are important since they have to be inside the brain borders and have to be defined separately. In this work, the regions have been defined automatically to overcome these drawbacks and to increase the efficiency of the method.

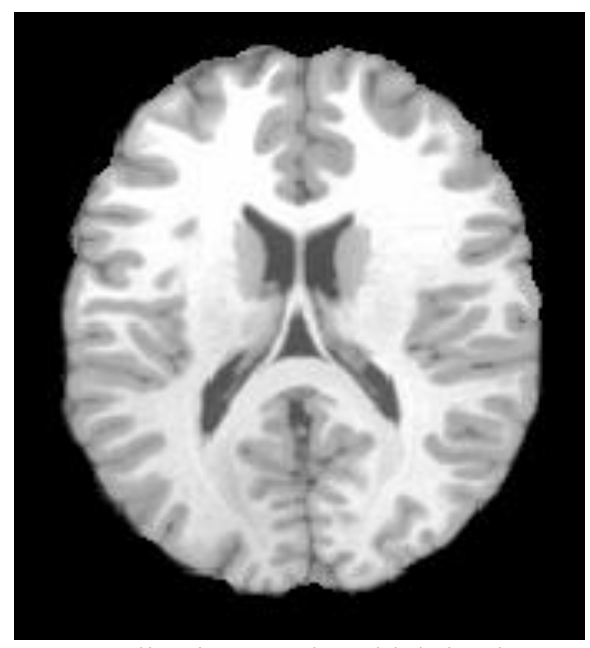

Figure 2. Normalization result, which is almost the same with the original image given in Figure 1, obtained by [23] using three regions that have size less than $20 \times 20$ pixels on a reference image

A recently published local normalization technique [24] seems efficient. Therefore, it has been applied to compare its results with the results of the proposed method. Also, the widely used methods (Gaussian and homomorphic filtering) have been applied to show their efficiency on the same images used in this work. Performances of these methods have been evaluated by comparing their results (Section 5). Contributions of this study can be given as: 1) Presentation of the state-of-the-art methods for intensity normalization in MR images. 2) Presentation of a full automatic and probabilistic method for brain MR image normalization. 3) Comparison of the results that have been obtained from four different intensity normalization approaches, which have been implemented in this work with the same MR images. The organization of the other sections of this paper is as follows. Intensity normalization methods applied in the literature are reviewed in Section 2. The normalization method [23], on which the work in this study is based, is explained in Section 3. The proposed FAIN algorithm is presented in Section 4. Results of four different intensity normalization approaches, which have been applied in this work, are given in Section 5. Finally, conclusions are presented in Section 6.

\section{Intensity Normalization Approaches in the Literature: A Survey}

Except the three works given in [25-27], which are not new and do not include the state-of-the-art techniques, according to our knowledge, there is not a survey type comprehensive work about intensity normalization techniques applied with MR images in the literature. Therefore, a survey, which can be useful for other researchers who are working in this area or interested in intensity normalization methods, is presented in this section. In addition to the methods given in the previous review works [25-27], several approaches have been proposed with different MR image datasets. For example, anatomical regions have been defined from an atlas and used to normalize intensity values in [28]. The normalization tool in [29] is based on a non-linear registration to register a brain atlas to the observed data. However, atlas based approaches may not always be efficient for an image, which is unlike those images available in the atlas.

In [30], intensity landmarks, which can be represented as $\mathrm{I}_{\mathrm{L}}=\left\{\mathrm{pc}_{1}, \mathrm{i}_{\mathrm{p} 10}, \mathrm{i}_{\mathrm{p} 20}, \ldots . \mathrm{i}_{\mathrm{p} 90}, \mathrm{pc}_{2}\right\}$ where $i_{\mathrm{p}}$ refers to the intensity at $l^{\text {th }}$ percentile, have been learned from a training set. The normalization is provided with transformation of the original gray level values between 2 landmarks to the learned landmarks. However, in this approach, normalization results may change depending on the defined training dataset. Histogram mapping has been applied for normalization using joint histogram [31]. It is based on matching the histogram of a reference image to the histogram of the input image. However, this method is unreliable for brain MR images [32]. Because, better prior knowledge based on the neighborhood is required [33]. However, this algorithm is based on a non-rigid registration method for histogram mapping, which also increases computational complexity and processing time.

In $[34,35]$, the integral of intensity values has been computed and the mean of the integral value has been used for normalization. Similarly, each image has been normalized individually in [36]. In this method, each voxel is set to the mean value of a set of the highest intensity voxels. This algorithm might not be efficient in case of the 
maximum intensity value in some images. Because those images may have peak gray level values due to noise. A statistical active contour based model, which provides both intensity normalization and image segmentation, has been applied in [37]. However, results obtained by this method are changing according to the first location of the active contour [38].

In the literature, intensity normalization is mostly performed by Gaussian filtering [10-14,39]. However, results obtained from an original image (Figure 3.a) by this method may not be efficient in case of a chosen small $\sigma$ value (Figure 3.b). If a large $\sigma$ value is chosen then edges between tissues and so valuable information may be lost (Figure 3.c). Therefore, the $\sigma$ parameter should be chosen carefully.
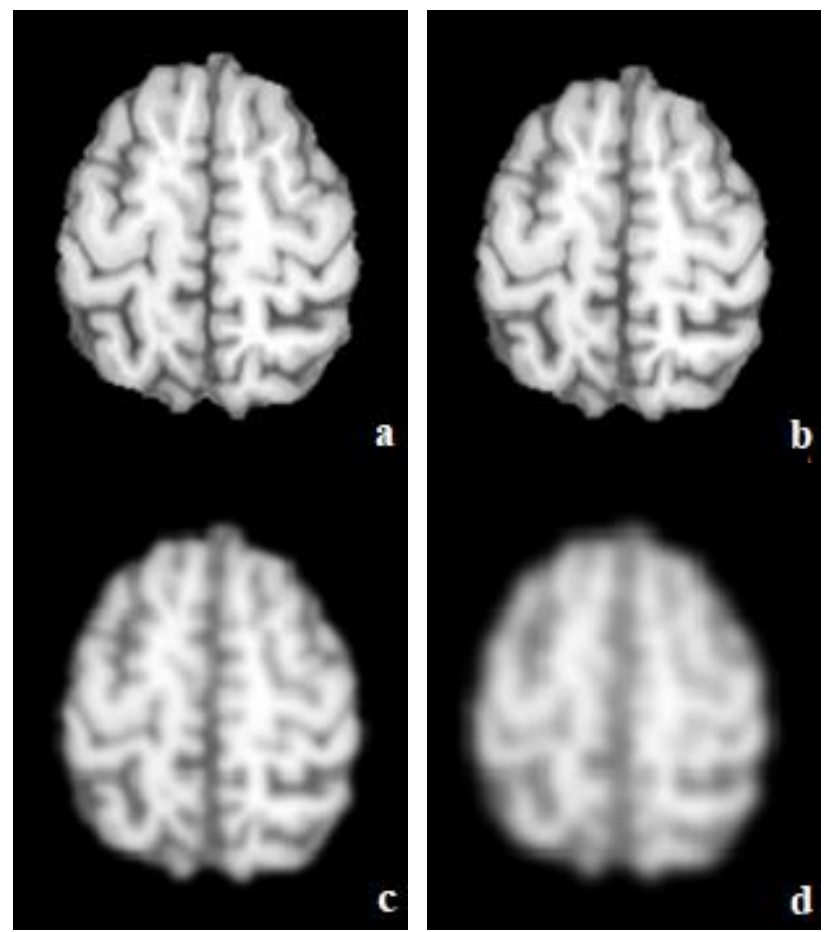

b

Figure 3. Original image (a); Image obtained by Gaussian filtering with $\sigma=0.5(\mathrm{~b}), \sigma=3$ (c) and $\sigma=5$ (d)

Another widely used normalization is homomorphic filtering [15-22]. Homomorphic filtering normalizes intensity values by removing multiplicative noise in images. The intensity value of a pixel can be defined as the product of the reflectance of the objects in the scene and the illumination of the scene. In other words, an image can be given with the following statement (i.e., with the illumination-reflectance model).

$$
I(x, y)=L(\mathrm{x}, \mathrm{y}) R(\mathrm{x}, \mathrm{y})
$$

In (1), the term $I(x, y)$ corresponds to the image, $R(x, y)$ corresponds to scene reflectance, $L(x, y)$ corresponds to scene illumination.

Illumination occurs due to the lighting conditions during image capturing. However, reflectance $R(x, y)$ results from the features of the objects themselves. When illumination and reflectance is compared, it can be observed that illumination changes very slowly within the image. However, reflectance may vary suddenly at boundaries of objects. Separation of reflectance and illumination components is based on this difference. The idea to remove the non-uniform illumination effect is to keep only the reflectance component while removing the illumination component. If illumination is considered as the noise that should be removed in the image, then this model becomes similar to the model given in Equation (1).

Homomorphic filtering is applied by transforming the multiplicative components to additive components with the following computations.

$$
\begin{gathered}
\ln (I(x, y))=\ln (L(\mathrm{x}, \mathrm{y}) R(\mathrm{x}, \mathrm{y})) \\
\ln (I(x, y))=\ln (L(\mathrm{x}, \mathrm{y}))+\ln (R(\mathrm{x}, \mathrm{y}))
\end{gathered}
$$

The next step in homomorphic filtering is to apply highpass filtering in logarithm domain to avoid the effect of the illumination component that has low-frequency. This process also keeps the reflectance component that has the high-frequency. Experimental results obtained by this method are presented in Section 5.

Recently, a local normalization technique [24] has been proposed to overcome two important problems in Gaussian filtering based normalization. The first problem in Gaussian filtering is that it is not clear how to choose the best $\sigma$ value for an image. The second problem is that it is not guarantee that the chosen $\sigma$ value will be best for remaining images in the dataset. Therefore, this technique in [20] uses a Sigma Adaptive Gaussian (SAG) filtering with the following equation.

$$
I_{N}(x, y)=\frac{I_{g}(x, y)-\mu(x, y)}{\sigma(x, y)}
$$

The term $I_{g}(x, y)$ in Equation (4) corresponds to the original grayscale image. $I_{N}(x, y)$ corresponds to the image obtained after normalization. The terms $\mu(x, y)$ and $\sigma(x, y)$ correspond to the mean and variance computed by Gaussian filtering on the image, $I_{g}(x, y)$. The $\sigma$ value for each image is obtained using the distance map that is 
generated by applying Euclidean distance transformation. Distance maps are constructed from binary images which are obtained from original grayscale images by adaptive thresholding given in [40]. The Euclidean transformation assigns a number, which is the distance value between that pixel and the nearest zero valued pixel in the binary image (It should be noted here that zero valued pixels correspond to background region and nonzero valued pixels correspond to foreground region in the binary image). If we assume $\max D$ as the maximum value in the distance map, the sigma parameter of the Gaussian filter is computed by,

$$
\sigma_{\text {Gauss }}=\min (T, \operatorname{maxD})
$$

where the threshold value $T(\mathrm{~T}=100)$ is a constant value and found experimentally. The reason to use the term $T$ and $\max D$ is to provide smoothing on the maximum valued pixels as much as possible and to avoid blurring effects (over-smoothing), while simultaneously smoothing. If the term $\max D$ is low then under-smoothing can occur. Therefore, to address both under- and over-smoothing cases, the sigma parameter of the Gaussian filter is computed by,

$$
\sigma_{\text {Gauss }}= \begin{cases}\max (t, \max D), & \text { if } \max D<t \\ \min (T, \max D), & \text { otherwise }\end{cases}
$$

where $t(\mathrm{t}=50)$ is chosen experimentally. The algorithm of this method is given in Table $1[24,41]$.

Table 1. Algorithm of the SAG filtering based local normalization method

1. Get a grayscale image $I_{g}$ and $\sigma_{\text {Gauss }}$

2. By assigning zero value to the $\sigma_{\text {Gauss }}$ and mean, compute the mean value $\mu_{\text {Gauss }}$ with cumulative distribution function

3. Obtain the first filtered image $\left(I_{f 1}\right)$ by applying Gaussian filtering with the grayscale image, $\sigma_{\text {Gauss }}$ and $\mu_{\text {Gauss }}$

4. Take difference between the grayscale image and filtered image ( $\left.I_{f 1}\right)$ to obtain an image, which is called as $I_{\text {Difference }}$ corresponding to the nominator in (4) (i.e., $I_{\text {Difference }}=I_{g}-I_{f 1}$ )

5. Generate a temporary image, $I_{\text {temp }}$, by computing squares of intensity values in the $I_{\text {Difference }}$ (i.e.,

$$
I_{\text {Temp }}=I_{\text {Difference }}{ }^{\wedge} \text { ) }
$$

6. Apply Gaussian filtering using the temporary image $I_{\text {Temp }}, \mu_{\text {Gauss }}$ and $\sigma_{\text {Gauss }}$ terms to generate the second filtered image $\left(I_{f 2}\right)$.

7. Compute the square root of all intensity values in the $I_{f 2}$ to obtain a new image, which is $I_{\text {Denominator }}$ and corresponds to the denominator in (4).

8. Obtain the normalized image by dividing intensity values in $I_{\text {Difference }}$ to the intensity values in $I_{\text {Denominator }}$.

This technique seems efficient on pathological images [24, 41]. To see its performance on brain MR images, it has been implemented and results have been presented (Section 5). In addition to the intensity normalization techniques given above, a novel approach proposed recently is based on varying distribution matching [23], on which the work in this study is based, is explained in the next section.

\section{Intensity Normalization Based on Varying Distribution Matching [23]}

Gray level values in MR images do not only depend on the underlying tissue types. These values also can change according to developmental processes, artifacts caused by scanners and disease progression. Therefore, normalization of intensity values should be based on spatially varying distribution matching. Because this approach brings an image into the coordinate system of a template using a coordinate transformation to make meaningful comparisons of spatially varying data. In a recently published work [23], a normalization method based on varying distribution matching has been implemented. It is a semi-automated method since it requires three different regions that are defined manually by users.

The first step in this method is identification of the userdefined regions (Figure 4.b,d) on an image, which is used as the reference image (Figure 4.a,c) that is selected by finding the most "typical" image in the dataset.
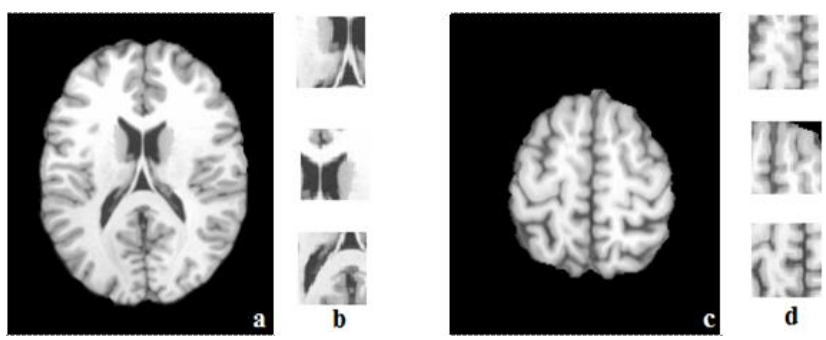

Figure 4. Example reference images $(\mathrm{a}, \mathrm{c})$ and user defined partitions $(b, d)[23]$ 
After definition of the three regions, mean gray level values are computed from each region for each tissue components (WM, GM, CSF). For this purpose, gray level values are fitted to Gaussian Mixture Model (GMM) [42]. After this step, 1) the average of the mean gray level values of the WM, GM and CSF tissue in those regions, and 2) the average of the mean gray level values of the brain tissues in the observed image are computed. By using these average values, a transfer (mapping) function is generated. In the final step, normalization of intensities in the observed image is performed by using the mapping function. The steps of this method are given in Figure 5 [23].

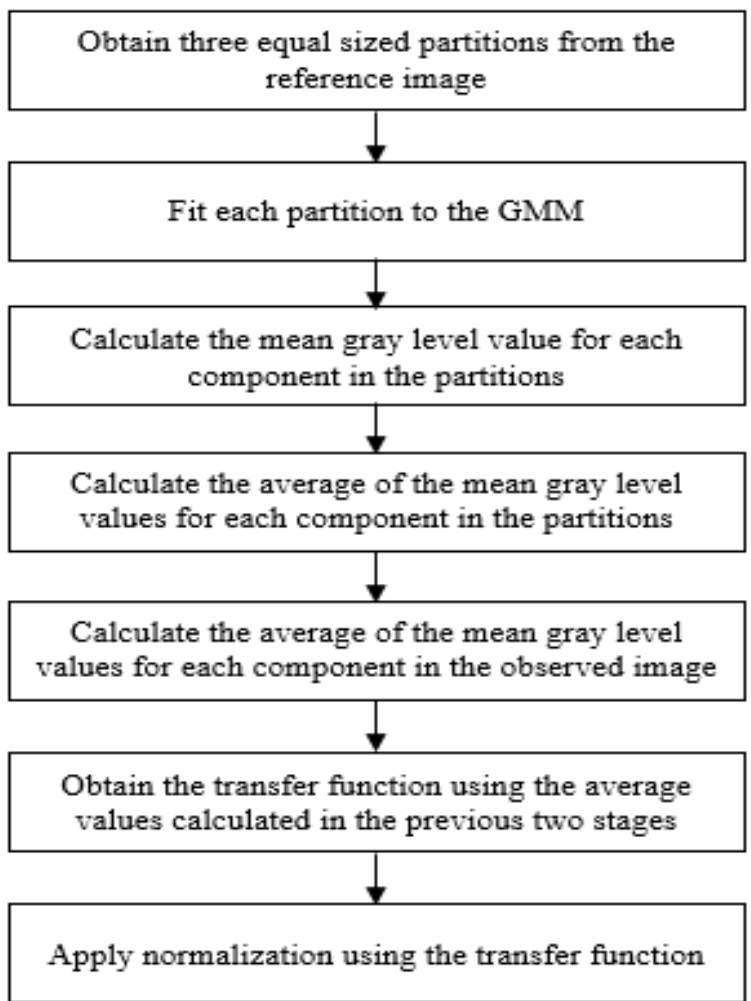

Figure 5. The steps of the intensity normalization based on varying distribution matching [23]

GMM mixtures three Gaussian models (for the three tissues; WM, GM, CSF) in the brain image. Expectation Maximization (EM) [43] has been applied to estimate the parameters of the GMM. To explain how these parameters are estimated iteratively with the EM approach, let $I$ is an image that can be defined as $I: U \rightarrow[0.1]^{c}$, where $U=$ $\llbracket 0 ; n-1 \rrbracket \times \llbracket 0 ; m-1 \rrbracket$ are pixels, the term $m$ is the number of rows, the term $n$ is the number of columns, and $c$ refers to the numbers of color channels, which are usually given as $c \in\{1,3\}$.

In GMM, image is modelled using the probability distribution that can be given with $I(i)$, which refers to $i$ th pixel in the image $I$, as

$$
P(I(i))=\sum_{k=1}^{K} w_{k} N\left(I(i) \mid \mu_{k}, \sigma_{k}^{2}\right)
$$

where the term $K$ corresponds to the number of clusters, $w_{k}$ is a positive weight value, $N\left(I(i) \mid \mu_{k}, \sigma_{k}^{2}\right)$ is the Gaussian function, the terms $\mu_{k}$ and $\sigma_{k}^{2}$ refer to mean and variance values for cluster $k$. The EM algorithm is performed with 1) initialization, 2) expectation, and 3) maximization steps.

In the initialization step, $w_{k}, \mu_{k}$, and $\sigma_{k}^{2}$ parameters are initialized by $\mathrm{k}$-means method for each cluster. K-means based clustering has been applied widely in different works $[44,47]$.

The main idea in the k-means algorithm is to find the best centers for $k$ clusters. Each center should be placed properly. Because different locations of these centers lead to different results. Therefore, the most appropriate choice is to place centers far away from each other. For this purpose, separation of data points into clusters is provided by minimization of the function that is a squared error and given as

$$
J=\sum_{j=1}^{k} \sum_{i=1}^{U}\left\|x_{i}^{(\mathrm{j})}-c_{j}\right\|^{2}
$$

where the term $\left\|x_{i}^{(\mathrm{j})}-c_{j}\right\|^{2}$ is the distance between cluster center $\left(c_{j}\right)$, which shows the distance of $\mathrm{n}$ data points from their cluster centers, and a data $x_{i}^{(\mathrm{j})}$.

In the expectation step, conditional probability $\mathrm{P}\left(\mathrm{C}_{k} \mid \mathrm{I}(i)\right)$ is computed for each cluster $\mathrm{C}_{\mathrm{k}}$ and pixel $I(i)$.

$$
P\left(C_{k} \mid I(i)\right)^{(t)}=\frac{w_{k}^{(\mathrm{t})} N\left(I(i) \mid \mu_{k}^{(t)} \sigma_{k}^{2(t)}\right)}{\sum_{j=1}^{K} w_{j}^{(\mathrm{t})} N\left(I(i) \mid \mu_{j}^{(t)} \sigma_{j}^{2(t)}\right)}
$$

In (2), $t$ refers the number of iterations. In this stage, a posterior probability is assigned to each possible association.

In the maximization step, the bound obtained in the previous stage is optimized to improve the estimate. In this step, $w_{k}^{(\mathrm{t})}, \mu_{k}^{(t)}$ and $\sigma_{k}^{2(t)}$ are maximized with probabilities $P\left(C_{\mathrm{k}} \mid I(\mathrm{i})\right)$ by the following statements; 


$$
\begin{gathered}
\mu_{k}^{(t+1)}=\frac{\sum_{i}^{U} P\left(C_{k} \mid I(i)\right)^{(t)} \cdot I(i)}{\sum_{i}^{U} P\left(C_{k} \mid I(i)\right)^{(t)}} \\
\sigma_{k}^{(t+1)}=\frac{\sum_{i}^{U} P\left(C_{k} \mid I(i)\right)^{(t)} \cdot\left(I(i)-\mu_{k}^{(t+1)}\right)^{2}}{\sum_{i}^{U} P\left(C_{k} \mid I(i)\right)^{(t)}} \\
w_{k}^{(t+1)}=\frac{\sum_{i}^{U} P\left(C_{k} \mid I(i)\right)^{(t)}}{U}
\end{gathered}
$$

The average of the mean gray level values in the regions and the average of the mean gray level values in a given image, whose intensity values will be normalized, are computed for each tissue. A mapping function is generated by using these average values. Normalization of intensity values in the given image is performed with the mapping function. Figure 6 shows the transfer functions (Figure 6.a) that have been generated with the chosen reference image (Figure 4.a) and an observed image (Figure 6.b) [23].

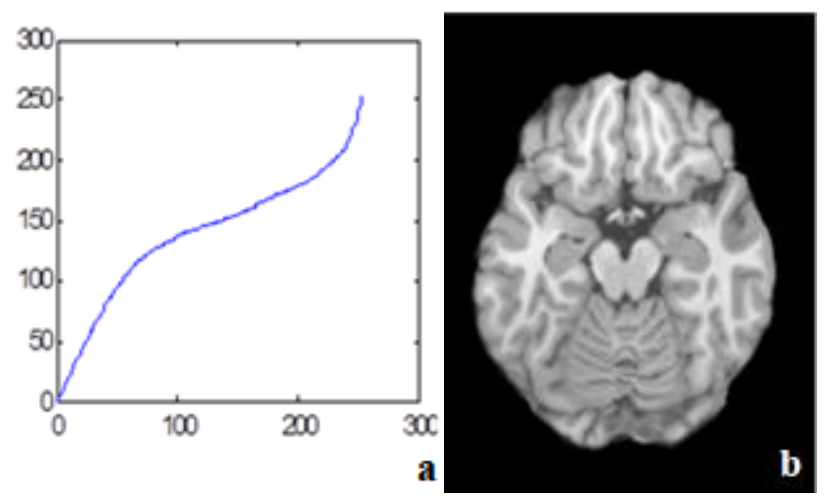

Figure 6. Transfer function (a); Observed (original) image (b)

However, the results obtained by this method depend on experience and attention of users. Because users may define less number of regions on some images due to their limited time and intensive works. Also, size of the regions may not be equal or large enough to get statistical information about brain tissues. Moreover, the regions may be defined at outside (or partially outside the brain) or as overlapping regions.

The number, size and locations of the user-defined regions are important factors that affect the performance of the method. Therefore, the regions should be defined carefully.

To overcome the problems of the semi-automated normalization method and to increase its efficiency, it has been improved (Section 4) in this study.

\section{The Proposed FAIN Method}

The proposed FAIN method uses statistical features of five regions that are defined automatically at different lobes inside the brain. Also, the size of each region is calculated automatically according to the vertical and horizontal length of the brain.

In this method, after choosing a reference image, it is clustered into two clusters, which correspond to foreground and background in the image, by k-means algorithm. Figure 7.a shows the foreground obtained by clustering of the reference image given in Figure 4.a.

The next step is to find boundaries (at the right, left, bottom and top side) of the brain to identify regions inside the brain. For this purpose, from the left, right, top and bottom side, the position of the first non-zero pixel (which is shown with blue and yellow colored lines in Figure 7.b) is found.

Then, the horizontal and vertical length of the brain is calculated. The region at the left-hand side is defined with $30 \%$ width (of the horizontal length of the brain) and $40 \%$ length (of the vertical length of the brain). The region should not include any background part (i.e., part including zero valued pixels close to boundaries in the binary image in Figure 7.a). Therefore, to be sure that the region includes only foreground part, the region is defined inside the brain by beginning from the position where $5 \%$ of the horizontal length of the brain.

Similarly, the regions at the right-hand side, top and bottom side are defined. In addition to these four regions, one more region is defined at the center to get more information about intensity values of each tissue. To define the region at the center, the intersection of the middle of the horizontal and vertical length is used. The rectangular region at the center is defined by beginning from the intersection point to the position (at the right- and left-hand side) at the distance corresponding to $15 \%$ of the horizontal length. The height of this region is defined similarly by beginning from the intersection point to the position (at the top and bottom side) at the distance corresponding to $20 \%$ of the vertical length. By this way, the region is obtained with $30 \%$ width and $40 \%$ height. All identified regions are shown with red colored rectangular shapes in Figure 7.b. To increase visibility of the three tissues in those regions, they are also shown after magnification in Figure 7.c. 

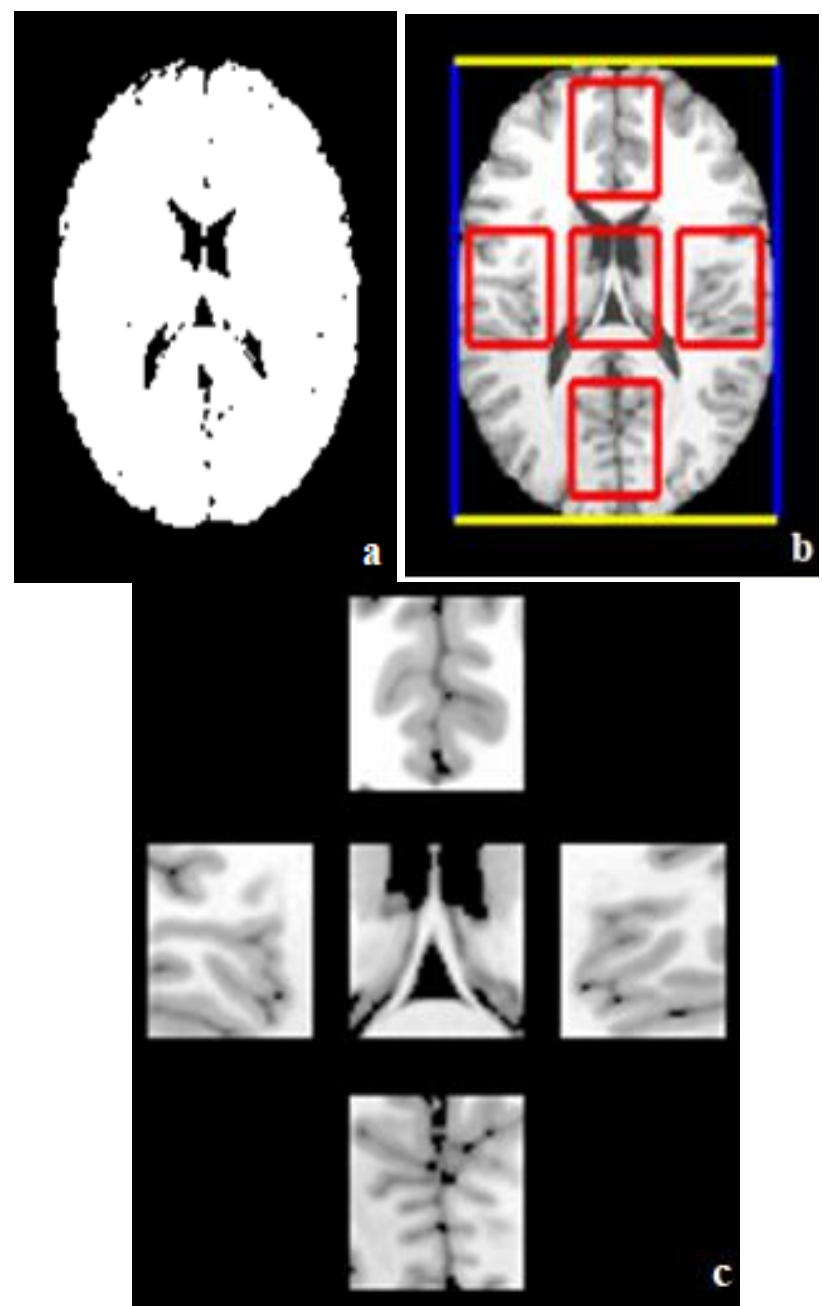

Figure 7. The foreground image obtained after clustering the reference image in Figure 4.a into two clusters (a); Brain borders (shown with yellow and blue colored lines) and identified five regions (shown with red colored rectangular shapes) (b); To increase visibility of the tissues in those regions, they are shown after magnification (c)

Then the mapping function is generated with average gray level values and intensity normalization is performed according to this function.

For an example, Figure 8.a shows the mapping function generated with the average of the mean gray level values obtained from the observed (original) image (Figure 8.b) and the five regions (Figure 7.b) in the reference image. The image obtained after this process is shown in Figure 8.c.

The efficiency of this approach is shown on the brain MR image data sets (Section 5). These images have been obtained from the database of the Laboratory of Neuro Imaging at the University of Southern California [48].
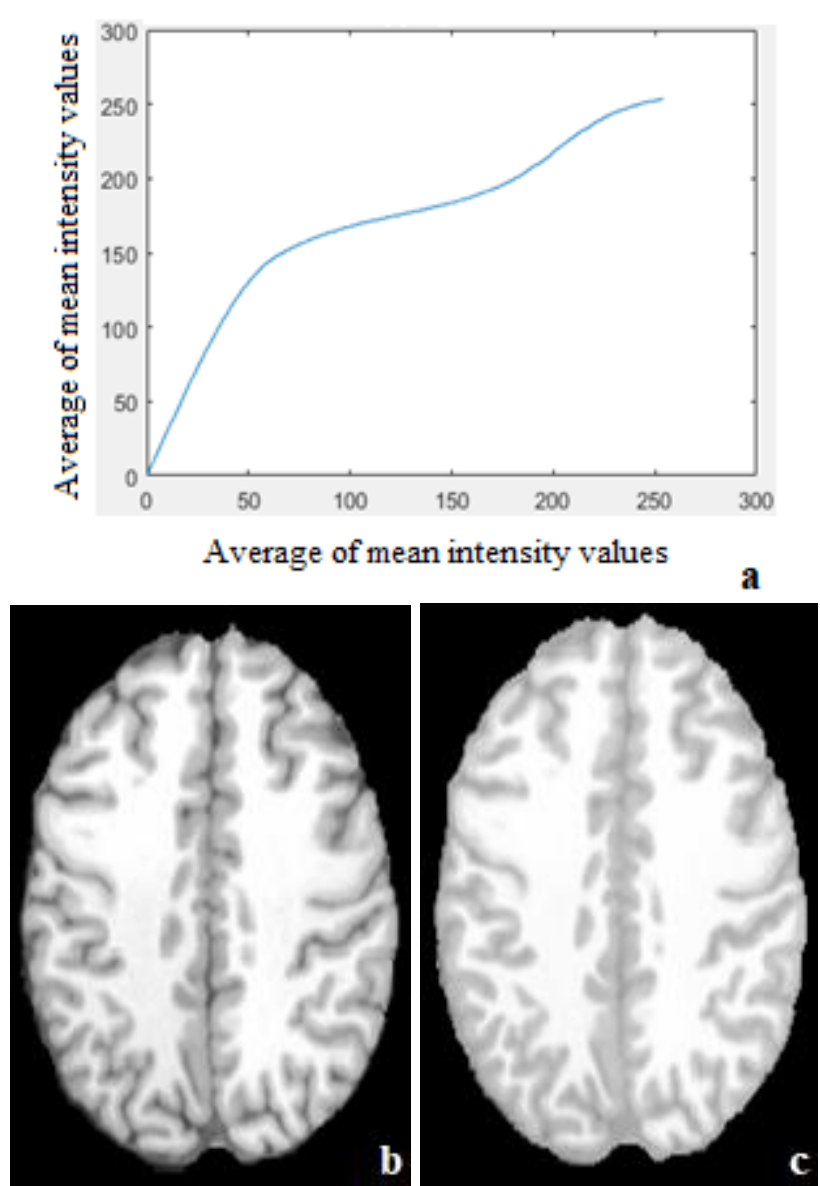

Figure 8. The mapping function (a); Observed image (b); The result obtained by the proposed FAIN approach (c)

\section{Results}

Experimental results of all methods implemented in this work are presented in Figure 9. Optimal parameters used in Gaussian and homomorphic filtering methods have been found experimentally. The $\sigma$ parameter for Gaussian filtering has been chosen as 2 , the cut-off frequency used in homomorphic normalization has been chosen as 0.5 .

\section{Conclusions}

In this paper, a survey on the-state-of-the art techniques about intensity normalization in MR images has been presented. Mostly applied normalization methods (Gaussian filtering and homomorphic filtering) and recently applied SAG filtering based local normalization have been implemented to show their performances on the same brain MR images. Also, a fully-automated and adaptive normalization method has been proposed for brain MR images. The results obtained from four different intensity normalization approaches have been presented to compare their effect on the same brain MR images. 


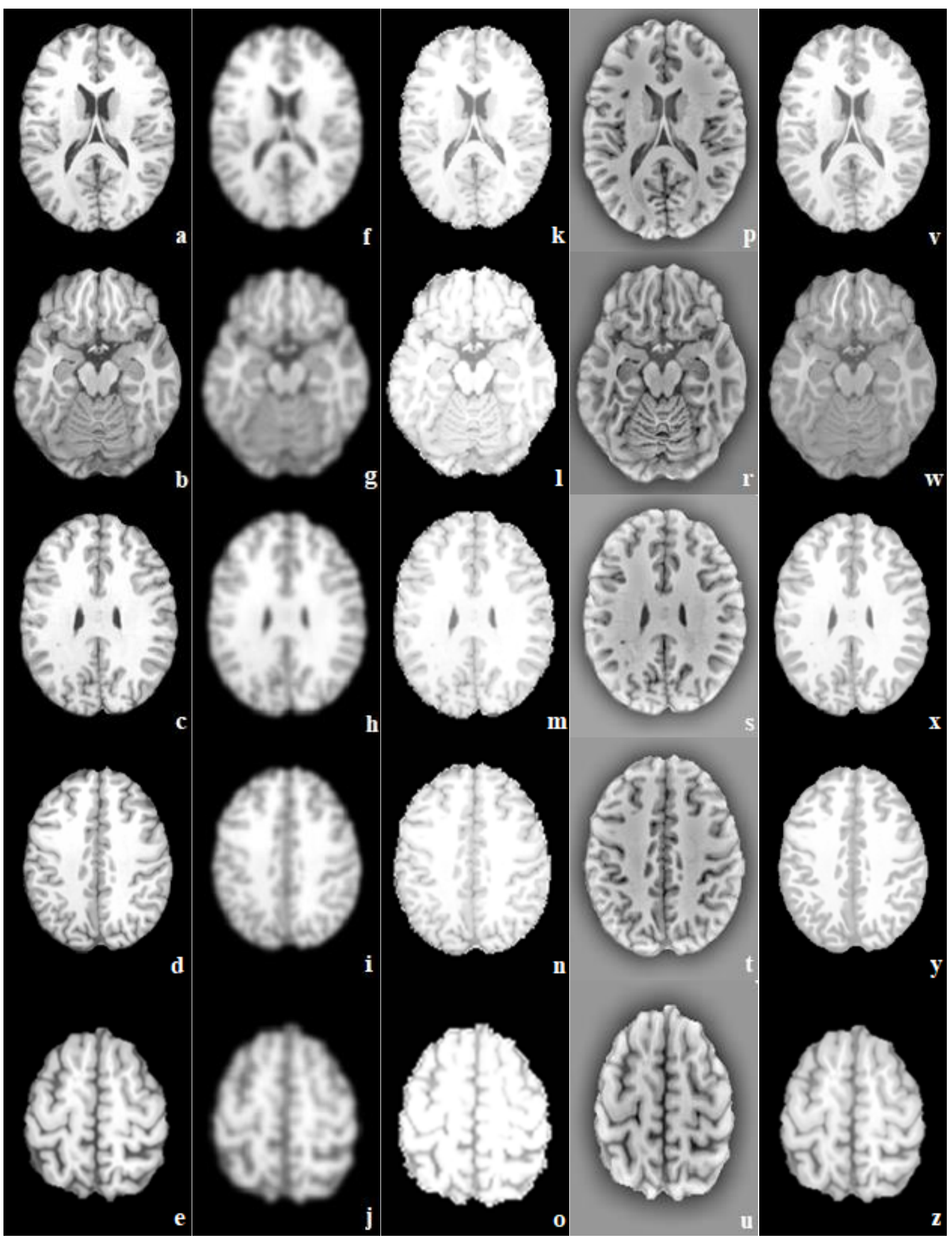

Figure 9. Original image (a); The result obtained by the Gaussian filtering (b), homomorphic filtering (c), local normalization with SAG filtering (d) and by the proposed FAIN method (e) 
It has been observed that although there are various approaches for intensity normalization in the literature, they have been applied with different types of images. Every image has its own characteristics. Therefore, the best normalization technique should be chosen according to the properties of images. Intensity normalization should be efficient and easy to implement. The results obtained by Gaussian and homomorphic filtering based normalization methods are sensitive to the chosen user-defined parameters. Semi-automated methods are flexible but may not be robust or reliable since they need user-defined parameters, which may not always be chosen as the most appropriate values.

The proposed normalization approach in this work uses tissue-specific intensity information since mean intensity values of each tissue are calculated separately. Properties of the proposed method are as follows. 1) There is no overlapping of the regions defined automatically on images. 2) Sizes of those regions are calculated adaptively for each image according to the horizontal and vertical length of the brain. 3) Region number (which is five) is always the same for each image. 4) Different lobes (e.g., left, right, bottom and top) of the brain are used to define regions. The proposed method can be applied with different medical images to see its performance. In this work, a model based classification has been used. Other classification methods, which can be developed according to properties of images, can be applied to increase the efficiency of the proposed approach. There is not real inhomogeneity field (in other words ground truth data). Therefore, objective evaluation is not possible. Visual evaluation of results is performed by taking into account some features such as the visibility of tissues and their boundaries. Comparisons of the results (Figure 9) obtained by the methods implemented in this work show that the proposed FAIN algorithm gives more efficient results on the brain MR images used in this study.

\section{References}

1. Hellier, P, Consistent intensity correction of MR images, In proceedings of the IEEE International Conference on Image Processing, (ICIP 2003), Barcelona, Spain, 2003, pp.1109-1112.

2. Sweeney, E.M, Shinohara, R.T, Shea, C.D, Reich, D.S, Crainiceanu. C.M, Automatic lesion incidence estimation and detection in multiple sclerosis using multisequence longitudinal MRI, American Journal of Neuroradiology, 2012, 34(1), 68-73.

3. Shah, M, Xiao, Y, Subbanna, M, Francis, S, Arnold, D.L, Collins, D.L, Arbel, T, Evaluating intensity normalization on MRIs of human brain with multiple sclerosis, Medical Image Analysis, 2011, 15(2), 267-282.

4. Meier, D.S, Guttmann, C.R.G, Time-series analysis of MRI intensity patterns in multiple sclerosis, NeuroImage, 2003, 20(2), 193-209.

5. Madabhushi, A, Udupa, J.K, Moonis, G, Comparing MR image intensity standardization against tissue characterizability of magnetization transfer ratio imaging, Journal of MagneticRresonance Imaging, 2006, 24(3), 667-675.
6. Loizou, C.P, Pantziaris, M, Seimenis, I, Pattichis, C.S, Brain MR image normalization in texture analysis of multiple sclerosis, In proceedings of the $9^{\text {th }}$ IEEE Conference on Information Technology and Applications in Biomedicine, Larnaca, Cyprus, 2009, pp.1-5.

7. Pourahmadi, M, Noorbaloochi, S, Multivariate time series analysis of neuroscience data: some challenges and opportunities, Current Opinion in Neurobiology, 2016, 37(1), pp. 12-15.

8. Jayender, J, Chikarmane, S, Jolesz, F.A, Gombos, E, Automatic segmentation of invasive breast carcinomas from dynamic contrastenhanced MRI using time series analysis, Journal of Magnetic Resonance Imaging, 2014, 40(2), 467-475.

9. Waarde, J.A, Scholte, H.S, Oudheusden, L.J.B, Verwey, B, Denys, D, Wingen, G.A, A functional MRI marker may predict the outcome of electroconvulsive therapy in severe and treatment-resistant depression, Molecular Psychiatry, 2015, 20, 609-614.

10. Kickingereder, P, Burth, S, Wick, A, Gotz, M, Eidel, O, Schlemmer, H.P, Maier-Hein, K.H, Wick, W, Bendszus, M, Radbruch, A, Bonekamp, D, Radiomic profiling of glioblastoma: identifying an imaging predictor of patient survival with improved performance over established clinical and radiologic risk models, Radiology, 2016, 280(3), 880-889.

11. Ellingson, B.M, Kim, H.J, Woodworth, D.C, Pope, W.B, Cloughesy, J.N, Harris, R.J, Lai, A, Nghiemphu, P.L, Cloughesy, T.F, Recurrent glioblastoma treated with bevacizumab: Contrast enhanced T1weighted subtraction maps improve tumor delineation and aid prediction of survival in a multicenter clinical trial, Radiology, 2013, 271(1), 200-210

12. Kickingereder, $P$, Radbruch, $A$, Burth, S, Wick, A, Heiland, $S$, Schlemmer, H.P, Wick, W, Bendszus, M, Bonekamp, D, MR perfusion-derived hemodynamic parametric response mapping of bevacizumab efficacy in recurrent glioblastoma, Radiology, 2016, 279(2), 542-552.

13. Newlander, S.M, Chu, A, Sinha, U.S, Lu, P.H, Bartzokis, G, Methodological improvements in voxel-based analysis of diffusion tensor images: Applications to study the impact of apolipoprotein E on white matter integrity. Journal of Magnetic Resonance Imaging, 2014, 39(1), 387-397.

14. Sarkka, S, Bayesian Filtering and Smoothing; Cambridge University Press: London, England, 2013; pp 252.

15. Fan, C.N, Zhang, F.Y, Homomorphic filtering based illumination normalization method for face recognition, Pattern Recognition Letters, 2011, 32, 1468-1479.

16. Dawoud, M, Altilar, D.T, Privacy-preserving search in data clouds using normalized homomorphic encryption, In proceedings of the Parallel Processing Workshops (Euro-Par 2014), Lecture Notes in Computer Science, 2014, 8806(1), 62-72.

17. Agarwal, T. K, Tiwari, M, Lamba, S. S, Modified histogram based contrast enhancement using homomorphic filtering for medical images, In preceeding of the IEEE International Conference on Advance Computing (IACC), India, 2014, pp.964-968.

18. Banik, R, Hasan, R, Iftekhar, S, Automatic detection, extraction and mapping of brain tumor from MRI scanned images using frequency emphasis homomorphic and cascaded hybrid filtering techniques, International Conference on Electrical Engineering and Information Communication Technology (ICEEICT), Dhaka, Bangladesh, 2015, pp.1-6.

19. Madhava, V, Yogesh, R, Srilatha, K, Wavelet decomposition on histogram based medical image contrast enhancement using 
homomorphic filtering. Biosciences Biotechnology Research Asia, 2014, 13(1), 457-462.

20. Agarwal, M, Mahajan, R, Medical images contrast enhancement using quad weighted histogram equalization with adaptive gama correction and homomorphic filtering, Procedia Computer Science, 2017, 115, 509-517.

21. Yang, D, Gach, H, Li, H, Mutic, S, TU-H-206-04: An effective homomorphic unsharp mask filtering method to correct intensity inhomogeneity in daily treatment MR images, The International Journal of Medical Physics Research and Practice, 2016, 43(6), 3774 .

22. Tan, Y, Li, G, Duan, H, Li, C, Enhancement of medical image details via wavelet homomorphic filtering transform, International Journal of Intelligent Systems, 2013, 23(1), 83-94.

23. Goceri, E, Intensity normalization in brain MR images using spatially varying distribution matching, In proceeding of the International Conference on Conferences Graphics, Visualization, Computer Vision and Image Processing (CGVCVIP 2017), Lisbon, Portugal, 2017, pp.300-304.

24. Goksel, B, Goceri, E, Elder, B, Puduvalli, V, Gurcan, M, Otero, J.J, Automated fluorescent miscroscopic image analysis of PTBP1 expression in glioma, Plos One, 12(3), e 0170991, 1-16.

25. Belaroussi, B, Milles, J, Carme, S, Zhu, Y.M, Benoit-Cattin, H, Intensity non-uniformity correction in MRI: Existing methods and their validation. Medical Image Analysis, 2006, 10(1), 234-246.

26. Vovk, U, Pernus, F, Likar, B, A review of methods for correction of intensity in-homogeneity in MRI. IEEE Transactions on Medical Imaging, 2007, 26(3), 405-421.

27. Likar, B, Viergever, M.A, Pernus, F, Retrospective correction of MR intensity in-homogeneity by information minimization, IEEE Transactions on Medical Imaging, 2001, 20(1), 1398-1410.

28. Velde, G.V, Rangarajan, J.R, Vreys, R, Guglielmetti, C, Dresselaers, T, Verhoye, M, Linden, A, Debyser, Z, Baekelandt, V, Maes, F, Himmelreich, U, Quantitative evaluation of MRI-based tracking of ferritin-labeled endogenous neural stem cell progeny in rodent brain, NeuroImage, 2012, 62, 367-380.

29. Souza, R, Lucena, O, Garrafa, J, Gobbi, D, Saluzzi, M, Appenzeller, S, Rittner, L, Frayne, R, Lotufo, R, An open, multi-vendor, multifield-strength brain MR dataset and analysis of publicly available skull stripping methods agreement, NeuroImage, 2017 (accepted), https://doi.org/10.1016/j.neuroimage.2017.08.021.

30. Pereira, S, Pinto, A, Alves, V, Silva, C.A, Brain tumor segmentation using convolutional neural networks in MRI images, IEEE Transactions on Medical Imaging, 2016, 35(5), 1240-1251.

31. Jager, F, Hornegger, J, Nonrigid registration of joint histograms for intensity standardization in magnetic resonance imaging. IEEE Transactions on Medical Imaging, 2009, 28(1), 137-50.

32. Verma, N, Cowperthwaite, M.C, Burnett, M.G, Markey, M.K, Image analysis techniques for the quantification of brain tumors on MR images. In: Suzuki, K. J (ed) Computational intelligence in biomedical imaging, Springer, New York, 2014, pp. 279-316.

33. Bergeest, J.P, Jäger, F, A comparison of five methods for signal intensity standardization in MRI. In: Tolxdorff, T, Braun, J, Deserno, T.M, Horsch, A, Handels, H, Meinzer, H-P (eds) Bildverarbeitung für die Medizin. Algorithmen - Systeme-Anwendungen Proceedings des Workshops, Springer, Berlin, 2008, pp. 36-40.

34. Friston, K. J, Ashburner, J. T, Kiebel, S. J, Nichols, T. E, Penny, W. D, Statistical parametric mapping: The analysis of functional brain images; Statistical Models and Experimental Design, Academic Press: London, UK, 2007, pp.656.

35. Illán, I.A, Górriz, J.M, Ramírez, J, Segovia, F, Jiménez-Hoyuela, J.M, Ortega-Lozano, S.J, Automatic assistance to Parkinson's disease diagnosis in DaTSCAN SPECT imaging, Medical Physics, 2012, 39(10), 5971-5980

36. Illán, I.A, Górriz, J.M, Ramírez, J, Salas-González, D, López, M.M, Segovia, F, Chaves, R, Gómez-Rio, M, Puntonet, C.G, 18F-FDG PET imaging analysis for computer aided Alzheimer's diagnosis, Information Sciences, 2011, 181(4), 903-916.

37. Zhang, K.H, Zhang, L, Lam, K.M, Zhang, D, A local active contour model for image segmentation with intensity inhomogeneity, 2013, arXiv:1305.7053, 1-27.

38. Liu, L.X, Zhang, Q, Wu, M, Li, W, Shang, F, Adaptive segmentation of magnetic resonance images with intensity inhomogeneity using level set method, Magnetic Resonance Imaging, 2013, 31(4), 567574.

39. Waarde, J. A, Scholte, H. S, Oudheusden, L. J. B, Verwey, B, Denys, D, Wingen, G.A, A functional MRI marker may predict the outcome of electroconvulsive therapy in severe and treatment-resistant depression, Molecular Psychiatry, 2015, 20, 609-614.

40. Feng, M.L, Tan, Y.P, Contrast adaptive binarization of low quality document images, IEICE Electron Express, 2004, 1(16), 501-506.

41. Goceri, E, Goksel, B, Elder, J. B, Puduvalli, V. K, Otero, J. J, Gurcan, M. N, Quantitative validation of anti-PTBP1 antibody for diagnostic neuropathology use: Image analysis approach, International Journal of Numerical Methods in Biomedical Engineering, 2017, e2862, 1-14.

42. Bishop, C.M, Mixture models and EM. In: Jordan, M, Kleinberg, J, Schölkopf, B, (editors) Pattern recognition and machine learning. Springer, New York, 2006, pp.423-455.

43. Dempster, A.P, Laird, N.M, Donald, B, Maximum likelihood from incomplete data via the EM algorithm, Paper presented at the Royal Statistical Society at a meeting organized by the Research Section, Atlantic City, N.J, 1977, pp.1-38.

44. Amorim, R.C, Makarenkov, V, Applying subclustering and Lp distance in weighted k-means with distributed centroids. Neurocomputing, 2016, 173(3), 700-707.

45. Ayech, M,W, Ziou, D, Terahertz image segmentation using k-means clustering based on weighted feature learning and random pixel sampling, Neurocomputing, 2016, 175(Part A), 243-264.

46. Sarrafzadeh, O, Dehnavi, A.M, Nucleus and cytoplasm segmentation in microscopic images using $\mathrm{K}$-means clustering and region growing. Journal of Advanced Biomedical Research, 2015, 4(1), 174-184.

47. Frandsen, P.B, Calcott, B, Mayer, C, Lanfear, R, Automatic selection of partitioning schemes for phylogenetic analyses using iterative kmeans clustering of site rates. BMC Evolutionary Biology, 2015, $15(1), 13-39$

48. University of Southern California, Brain Image Database. http://loni.usc.edu 2009 (accessed 25.10.2017). 